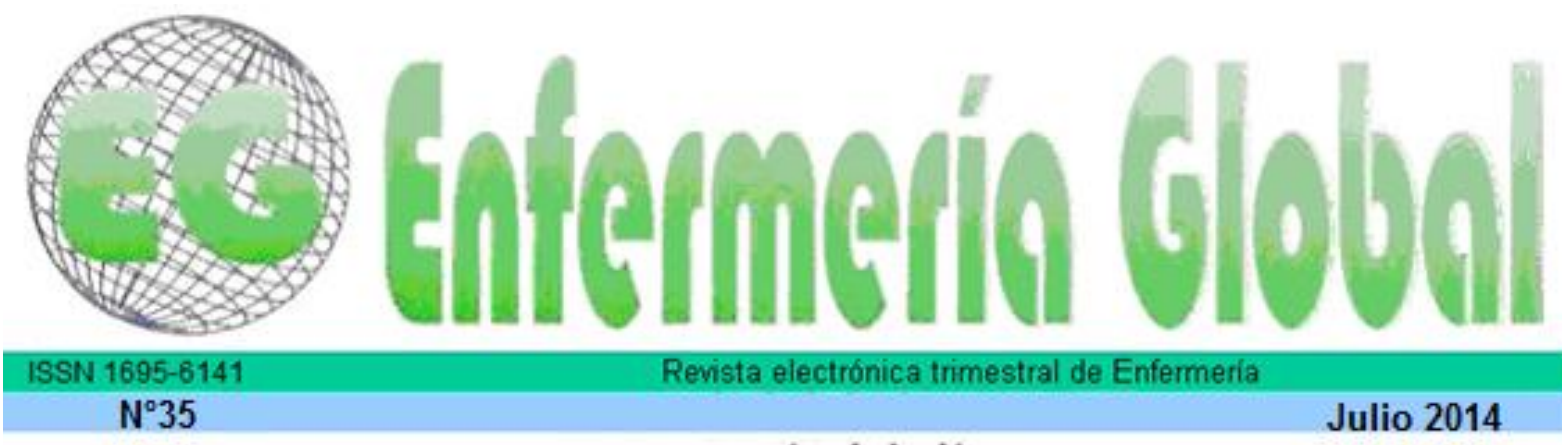

www.um.es/egloball

\title{
REVISIONES
}

\section{Implicaciones de la seguridad del paciente en la práctica del cuidado de enfermería}

Implicações da segurança do paciente na prática do cuidado de enfermagem,

Implications of patient safety in the practice of nursing care

\section{*Lima, Fabiane da Silva Severino *Souza, Natalia Pimentel Gomes **Freire de Vasconcelos, Patricia ${ }^{* * *}$ Aires de Freitas, Consuelo Helena ${ }^{* * * *}$ Bessa Jorge, Maria Salete ${ }^{* * * * *}$ De Souza Oliveira, Adriana Catarina}

*Alumna del Programa de Posgraduación Cuidados Clínicos en Enfermería y Salud de la Universidad Estadual de Ceará. E-mail: enfa.fabianelima@gmail.com ** Doctoranda del Programa de Posgraduación Cuidados Clínicos en Enfermería y Salud de la Universidad Estadual de Ceará. ***Doctora y Docente del Programa Cuidados Clínicos en Salud y Enfermería - Área de Concentración en Enfermería de la UECE. ****Enfermera. Profesora Titular de la UECE. *****Doctora en Enfermería. Docente en Enfermería - Gestión de los Servicios Sanitarios y Legislación Enfemera y del Programa de Máster y Doctorado en Ciencias Socio Sanitarias. Coordinadora del Trabajo Fin de Grado de Enfermería. Universidad Católica de San Antonio. Murcia.

Palabras clave: enfermería; seguridad del paciente; cuidados de enfermería

Palavras-chave: enfermagem; segurança do paciente; cuidados de enfermagem

Keywords: nursing; patient safety; nursing care. .

\section{RESUMEN}

El término seguridad viene siendo cada vez más discutido en el ámbito sanitario. Estando la Enfermería implicada en este proceso como promotora directa de acciones de seguridad por medio de sus prácticas de cuidado.

Este trabajo tiene como objetivo investigar cómo el término seguridad viene siendo abordado en el escenario del cuidado de enfermería en el ámbito nacional e internacional.

Investigación bibliográfica basada en artículos científicos publicados en revistas nacionales e internacionales indexadas en bases de datos Scientific Library (Scielo), Medical Literature Analysis and 
Retrieval System Online (MEDLINE) y SCOPUS, y publicadas en el período de 2007 a 2012. La recogida de datos ocurrió en el período de octubre de 2012. En total, fueron seleccionados 24 artículos, publicados en la lengua portuguesa, inglesa y española. Los fallos relatados con comprometimiento de seguridad del paciente fueron: en la comunicación oral o escrita entre profesionales del equipo y entre profesionales y paciente; terapéutica medicamentosa; realización de procedimientos de salud; y factores humanos relacionados al trabajo, como cansancio, falta de motivación, sobrecarga y/o (in)satisfacción. Como promotores de seguridad están: cuidado centrado en el paciente/familia, comunicación y diálogo, comunicación abierta sobre error; promoción de políticas de prevención, medición y evaluación de los errores y estandarización de las prácticas.

La enfermería desempeña un papel crucial en la promoción de la seguridad del paciente por presentarse de forma más constante y directa en el cuidado al paciente. En contrapartida, si dicho cuidado no es realizado con calidad, puede ser generador de errores y comprometer la seguridad del cuidado.

\section{RESUMO}

O termo segurança vem sendo cada vez mais discutido no âmbito sanitario. Estando a enfermagem implicada nesse processo como promotora direta de ações de segurança por meio de suas práticas de cuidado.

Este trabalho objetivou-se investigar como o termo segurança vem sendo abordado no cenário do cuidado de enfermagem no âmbito nacional e internacional.

Trata-se de uma revisão integrativa baseada em artigos científicos publicados em periódicos nacionais e internacionais indexados nas bases de dados Scientific Library (Scielo), Medical Literature Analysis and Retrieval System Online (MEDLINE) e SCOPUS e publicados no período de 2007 a 2012. A coleta de dados ocorreu no período de outubro de 2012. No total, foram selecionados 24 artigos, publicados na língua portuguesa, inglesa e espanhola. As falhas relatadas com comprometimento da segurança do paciente, foram: na comunicação oral ou escrita entre profissionais da equipe e entre profissional e paciente; terapêutica medicamentosa; realização de procedimentos de saúde; e fatores humanos relacionados ao trabalho, como cansaço, falta de motivação, sobrecarga e/ou (in)satisfação. Como promotores de segurança estão: cuidado centrado no paciente/família, comunicação e diálogo, comunicação aberta sobre o erro; promoção de gestão de pessoas; melhoria do sistema de prestação da assistência sanitária; promoção de políticas de prevenção, medição e avaliação dos erros e uniformização das práticas.

A enfermagem desempenha um papel crucial na promoção da segurança do paciente por apresentarse de forma mais constate e direta no cuidado ao paciente. Em contrapartida, se esse cuidado não for realizado com qualidade, pode ser gerador de erros e comprometer a segurança do cuidado.

\section{ABSTRACT}

The term safety is being increasingly discussed in the sanitary fields. Nursery is involved in this process as a direct promoter of security actions through their care practices.

This study aimed to investigate how the term safety has been addressed in the national and international scenario of nursing care.

Literature search based on scientific papers published in national and international journals indexed in databases Scientific Library (SciELO), Medical Literature Analysis and Retrieval System Online (MEDLINE) and SCOPUS published from 2007 to 2012. Data collection occurred in October 2012. In total, we selected 24 articles published in Portuguese, English and Spanish. The reported failures with compromised patient safety, were on oral or written communication between health professionals and between professionals and patients; drug therapy; procedures of health, and human factors related to work, such as fatigue, lack of motivation, overload and / or (dis) satisfaction. As promoters of safety are: patient-centered care / family communication and dialogue, open communication about the error; promotion of personnel management; improvement of the delivery system of health care, promoting prevention policies, measurement and evaluation of errors and standardization of practices. 
Nursing plays a crucial role in promoting patient safety by presenting up and finding more direct patient care. However, if this is not done carefully, it may generate errors and compromise the safety of care

\section{INTRODUCCIÓN}

A partir del final del siglo XX, el énfasis en la evaluación de la calidad de los servicios de salud se ha vuelto más importante respecto a la calidad de los servicios de salud, debido al aumento de las demandas de salud, con el consecuente aumento de los costes y limitación de recursos y un mayor esclarecimiento y, por tanto, mayor exigencia por parte de los usuarios del servicio ${ }^{(1)}$.

Así, se destacó la búsqueda de la calidad de los servicios de salud. Campbell, Roland et al (2000) ${ }^{(1)}$ entienden que la calidad expresada en términos de cuidado individual se representa cuando la persona tiene acceso al servicio de salud y al proceso de cuidar que necesitan.

Se entiende el acceso como elemento de calidad de los servicios de salud. Además, otros elementos son apuntados como imprescindibles para el alcance de la calidad, como la competencia del ejercicio profesional, disponibilidad y uso eficiente de recursos, promoción de la seguridad, satisfacción del usuario y resultados favorables para la salud de la población que está siendo asistida ${ }^{(2)}$.

Adoptamos, en este estudio, la definición de Seguridad propuesta por Terol y Agra ${ }^{(3)}$, que definen el término como la ausencia de daños producidos por el servicio de salud, así como estrategias destinadas a la prevención o minimización de los mismos.

La aplicación del término seguridad en la asistencia a la salud se justifica debido a la complejidad de la práctica clínica y la organización de la asistencia; su aplicación está fundamentada en las consecuencias que repercuten en la salud y en el bienestar de las personas que utilizan la asistencia para la salud ${ }^{(4)}$.

Sin embargo, una de las dificultades para abordar los problemas de seguridad es la creencia de que los errores derivados de la práctica cínica sean consecuencia solamente de acciones individuales. Los errores se derivan de todo el proceso asistencial $^{(5)}$

Forman parte de este proceso asistencial, tanto los profesionales de salud, los recursos disponibles y la gestión del proceso de trabajo. Entre los profesionales involucrados en la asistencia, está el equipo de enfermería que actúa de forma directa en el proceso de cuidado a la persona que está haciendo uso del servicio de salud.

El término Seguridad del paciente viene siendo adoptado desde el siglo XX por los profesionales que trabajan en la atención en salud. Los profesionales de enfermería están involucrados en este ramo por ser una categoría profesional que corresponde a mayor porcentaje de profesionales en el servicio y por desempeñar acciones de cuidado, tanto directo como indirecto, que pueden estar asociadas a la presencia de riesgos para la salud.

Por tanto, en el presente estudio, se optó por investigar cómo el término seguridad viene siendo abordado en el escenario del cuidado de enfermería en el ámbito nacional e internacional. 


\section{METODOLOGÍA}

Se trata de una revisión integrativa basada en artículos científicos publicados en periódicos nacionales e internacionales indexados en las bases de datos Scientific Library (Scielo), Medical Literature Analysis and Retrieval System Online (MEDLINE) y SCOPUS y publicados en el período de 2007 a 2012.

La elección de las referidas bases de datos se dio por tratarse de bibliotecas electrónicas que tenían vasta colección de periódicos brasileños e internacionales disponibles, muchas veces en forma de textos completos y on-line.

La colecta de artículos ocurrió en el período de octubre de 2012. Se utilizaron los descriptores "Seguridad del Paciente" y "Enfermería", debidamente registrados en el DECs (Descriptores en Ciencias de la Salud). La combinación de los descriptores resultó en 54 publicaciones. De estas se seleccionaron aquellas que estaban disponibles en su totalidad en Internet, por un total de 28 artículos. A continuación, se realizó la lectura de los resúmenes y seleccionados aquellos que presentaban respuestas al objetivo del presente estudio. Fueron excluídos los estudios de revisión bibliográfica y las producciones que no tenían el término "segurança", "safety" o "seguridad" en el resumen o descriptor de cada publicación, seleccionándose 24 artículos para análisis, siendo estos publicados en lengua portuguesa, inglesa y española.

\section{RESULTADOS}

La lectura de las obras seleccionadas culminó en incautaciones diversas acerca de la seguridad relacionada con los cuidados de enfermería. En la mayoría de los estudios, el término seguridad fue abordado de forma genérica, sin un mayor detalle de su definición.

En el cuadro I consta una presentación general de las publicaciones, identificándolas al lado por un código identificador que será utilizado para la referencia de las obras a lo largo de la presentación de los resultados. El cuadro I muestra que la mayoría de las obras se obtuvieron en el año 2012, con 11 (once) publicaciones. La lengua predominante fue la inglesa, con 20 (veinte) obras. Los objetivos listados en las publicaciones aparecen con abordajes diferentes del término seguridad en el ámbito del cuidado de enfermería. 
Cuadro 1: Presentación de las publicaciones identificadas en las bases de datos Scopus, Medline y Scielo por periódico, año, lengua, autor y objetivo de estudio. Fortaleza, Ceará, 2012.

\begin{tabular}{|c|c|c|c|c|c|c|}
\hline Código & Artículo & Periódico & Año & Lingua & Autor & Objetivo \\
\hline I & $\begin{array}{l}\text { Seguridad de los } \\
\text { pacientes. Un } \\
\text { compromiso de } \\
\text { todos para un } \\
\text { cuidado de } \\
\text { calidad }\end{array}$ & $\begin{array}{l}\text { Salud Uninorte. } \\
\text { Barranquilla } \\
\text { (Col.) }\end{array}$ & 2007 & $\mathrm{E}^{1}$ & $\begin{array}{l}\text { VILLARREAL } \\
\text { CANTILLO, } \\
\text { Elizabeth }\end{array}$ & $\begin{array}{l}\text { Reflexionar sobre la dimensión } \\
\text { del problema derivado de los } \\
\text { eventos adversos, para evitar su } \\
\text { ocurrencia y, así, promover } \\
\text { acciones para garantzair la } \\
\text { práctica segura. }\end{array}$ \\
\hline II & $\begin{array}{lr}\text { Redesenho } & \text { de } \\
\text { atividades } & \text { da } \\
\text { enfermagem } & \\
\text { para redução } & \text { de } \\
\text { erros } & \text { de } \\
\text { medicação } & \text { em } \\
\text { pediatria } & \end{array}$ & $\begin{array}{ll}\text { Rev } & \text { Bras } \\
\text { Enferm } & \end{array}$ & 2007 & $\mathrm{P}^{2}$ & $\begin{array}{l}\text { YAMANAKA, } \\
\text { Tatiana Inglez; } \\
\text { PEREIRA, } \\
\text { Daniela G.; } \\
\text { PEDREIRA, } \\
\text { Mavilde L. G. } \\
\text { and } \\
\text { PETERLINI, } \\
\text { Maria Angélica } \\
\text { S. }\end{array}$ & $\begin{array}{l}\text { Verificar la influencia del } \\
\text { rediseño de actividades de } \\
\text { enfermería para la } \\
\text { reducción de errores de } \\
\text { medicación en tres unidades de } \\
\text { pediatría de un hospital } \\
\text { universitario. }\end{array}$ \\
\hline III & $\begin{array}{lr}\text { Quality } & \text { and } \\
\text { strength } & \text { of } \\
\text { patient } & \text { safety } \\
\text { climate } & \text { on } \\
\text { medical-surgical } \\
\text { units }\end{array}$ & $\begin{array}{l}\text { Reprinted with } \\
\text { permission } \\
\text { from Health } \\
\text { Care } \\
\text { Management } \\
\text { Review, }\end{array}$ & 2009 & $\mathrm{I}^{3}$ & $\begin{array}{l}\text { HUGHES, Linda } \\
\text { C. } \\
\text { Yunkyung } \\
\text { CHANG, } \\
\text { Barbara A. Mark }\end{array}$ & $\begin{array}{l}\text { Describir el clima de seguridad } \\
\text { del paciente percibido por los } \\
\text { enfermeros, que actuan como } \\
\text { cuidadores directos en unidades } \\
\text { de enfermería médico- } \\
\text { quirúrgicas, seleccionados a } \\
\text { partir de una muestra nacional } \\
\text { de hospitales de cuidados } \\
\text { agudos. }\end{array}$ \\
\hline IV & $\begin{array}{l}\text { Hospitalized } \\
\text { Children's } \\
\text { Views of the } \\
\text { Good Nurse }\end{array}$ & Nursing Ethics & 2009 & I & Mary Brady & $\begin{array}{l}\text { Explorar la comprensión de } \\
\text { buena enfermera a partir de la } \\
\text { perspectiva del niño } \\
\text { hospitalizado. }\end{array}$ \\
\hline $\mathrm{V}$ & $\begin{array}{l}\text { "Universal } \\
\text { Precautions", } \\
\text { Perinatal Touch } \\
\text { and Examination } \\
\text { after Childhood } \\
\text { Sexual Abuse }\end{array}$ & Birth. & 2009 & I & Coles J, Jones K. & $\begin{array}{l}\text { Explorar las respuestas de las } \\
\text { mujeres en el contacto } \\
\text { profesional perinatal y examen } \\
\text { de ellas mismas y de sus bebês. }\end{array}$ \\
\hline VI & $\begin{array}{l}\text { Managing pain } \\
\text { using heat and } \\
\text { cold therapy }\end{array}$ & Paediatr Nurs & 2009 & I & $\begin{array}{l}\text { Lane E, Latham } \\
\text { T. }\end{array}$ & $\begin{array}{l}\text { Desarrollar evidencias para el } \\
\text { uso de calentamiento } \\
\text { superficial y refrigeración de } \\
\text { tejidos para proporcionar alivio } \\
\text { del dolor en niveles bajos a } \\
\text { moderados de dolor agudo en } \\
\text { niños en todo el Reino Unido. }\end{array}$ \\
\hline VII & $\begin{array}{l}\text { Keeping an eye } \\
\text { on patient safety } \\
\text { using human } \\
\text { factors } \\
\text { engineering } \\
\text { (HFE): a family } \\
\text { affair for the } \\
\text { hospitalized } \\
\text { child }\end{array}$ & $\begin{array}{l}\text { J Spec Pediatr } \\
\text { Nurs. }\end{array}$ & 2010 & I & $\begin{array}{l}\text { Barbara } \\
\text { Wilson }\end{array}$ & $\begin{array}{l}\text { Reflexionar acerca del cuidado } \\
\text { centrado en la familia, que } \\
\text { proporciona un fórum para } \\
\text { intercambio de informaciones } \\
\text { sobre los componentes básicos } \\
\text { de atención a niños y familias, } \\
\text { incluyendo el respeto, el } \\
\text { intercambio de información, } \\
\text { apoyo, colaboración y } \\
\text { construcción de confianza. }\end{array}$ \\
\hline VIII & $\begin{array}{l}\text { Safety is an } \\
\text { interpretive act: } \\
\text { A hermeneutic } \\
\text { analysis of care } \\
\text { in childbirth }\end{array}$ & $\begin{array}{l}\text { International } \\
\text { Journal of } \\
\text { Nursing } \\
\text { Studies }\end{array}$ & 2010 & I & $\begin{array}{l}\text { Elizabeth } \\
\text { Smythe }\end{array}$ & $\begin{array}{l}\text { Explorar la naturaleza } \\
\text { interpretativa de la seguridad a } \\
\text { través de un análisis } \\
\text { hermenéutico de las historias de } \\
\text { las mujeres que se sienten } \\
\text { inseguras. }\end{array}$ \\
\hline
\end{tabular}




\begin{tabular}{|c|c|c|c|c|c|c|}
\hline IX & $\begin{array}{lr}\text { Scales } & \text { for } \\
\text { assessing } & \text { self- } \\
\text { efficacy } & \text { of } \\
\text { nurses } & \text { and } \\
\text { assistants } & \text { for } \\
\text { preventing falls }\end{array}$ & J Adv Nurs. & 2010 & I & $\begin{array}{lr}\text { Dykes } & \text { PC, } \\
\text { Carroll } & \mathrm{D}, \\
\text { McColgan } & \mathrm{K}, \\
\text { Hurley } & \mathrm{AC}, \\
\text { Lipsitz } & \mathrm{SR}, \\
\text { Colombo } & \mathrm{L}, \\
\text { Zuyev } & \mathrm{L}, \\
\text { Middleton B. } & \\
\end{array}$ & $\begin{array}{l}\text { Relatar el desarrollo del test de } \\
\text { auto-eficacia para prevención } \\
\text { de caídas en la escala da } \\
\text { asistencia de enfermería. }\end{array}$ \\
\hline $\mathrm{X}$ & $\begin{array}{l}\text { Breastfeeding in } \\
\text { breast cancer } \\
\text { survivors: } \\
\text { pattern, } \\
\text { behaviour and } \\
\text { effect on breast } \\
\text { cancer outcome. }\end{array}$ & Breast. & 2010 & I & $\begin{array}{lr}\text { Azim HA } & \text { Jr, } \\
\text { Bellettini } & \text { G, } \\
\text { Liptrott } & \text { SJ, } \\
\text { Armeni } & \text { ME, } \\
\text { Dell'Acqua } & \text { V, } \\
\text { Torti F, } & \text { Di } \\
\text { Nubila } & \text { B, } \\
\text { Galimberti } & \text { V, } \\
\text { Peccatori F. } & \end{array}$ & $\begin{array}{l}\text { Analizar los comportamientos } \\
\text { de las pacientes con cáncer de } \\
\text { mama que habían iniciado la } \\
\text { lactancia y su efecto sobre el } \\
\text { resultado del cáncer de mama. }\end{array}$ \\
\hline XI & $\begin{array}{lr}\text { Revelação } & \text { da } \\
\text { ocorrência } & \text { de } \\
\text { erro } & \text { de } \\
\text { medicação } & \text { em } \\
\text { unidade } & \text { de } \\
\text { cuidados } & \\
\text { intensivos } & \\
\text { pediátricos } & \end{array}$ & $\begin{array}{ll}\text { Rev Bras } & \text { Ter } \\
\text { Intensiva } & \end{array}$ & 2010 & $\mathrm{P}$ & $\begin{array}{l}\text { Aline Santa Cruz } \\
\text { Belela, Maria } \\
\text { Angélica Sorgini } \\
\text { Peterlini, } \\
\text { Mavilde da Luz } \\
\text { Gonçalves } \\
\text { Pedreira }\end{array}$ & $\begin{array}{l}\text { Describir la ocurrencia de } \\
\text { comunicación del error de } \\
\text { medicación al equipo y familia } \\
\text { en una unidad de cuidados } \\
\text { intensivos pediátricos para } \\
\text { atención de pacientes } \\
\text { oncológicos. }\end{array}$ \\
\hline XII & $\begin{array}{l}\text { Capacitação de } \\
\text { enfermeiros para } \\
\text { uso da } \\
\text { ultrassonografia } \\
\text { na punção } \\
\text { intravascular } \\
\text { periférica }\end{array}$ & $\begin{array}{ll}\text { Acta } & \text { Paul } \\
\text { Enferm } & \end{array}$ & 2010 & $\mathrm{P}$ & $\begin{array}{l}\text { Ariane Ferreira } \\
\text { Machado Avelar; } \\
\text { Maria Angelica } \\
\text { Sorgini Peterlini; } \\
\text { Priscilla Sete de } \\
\text { Carvalho Onofre; } \\
\text { Myriam } \\
\text { Aparecida } \\
\text { Mandetta } \\
\text { Pettengill; } \\
\text { Mavilde da Luz } \\
\text { Gonçalves } \\
\text { Pedreira. }\end{array}$ & $\begin{array}{l}\text { Relatar la estrategia de } \\
\text { capacitación de enfermeros para } \\
\text { uso de la ultrasonografia } \\
\text { vascular en la obtención del } \\
\text { acceso vascular periférico. }\end{array}$ \\
\hline XIII & $\begin{array}{l}\text { The } \\
\text { Normalization of } \\
\text { Deviance: A } \\
\text { Threat to Patient } \\
\text { Safety }\end{array}$ & $\begin{array}{l}\text { J Perianesth } \\
\text { Nurs. }\end{array}$ & 2011 & I & $\begin{array}{ll}\text { Jan } & \text { Odom- } \\
\text { Forren } & \end{array}$ & No identificado. \\
\hline XIV & $\begin{array}{l}\text { TeamSTEPPS : } \\
\text { The Patient } \\
\text { Safety Tool That } \\
\text { Needs to Be } \\
\text { Implemented }\end{array}$ & $\begin{array}{l}\text { Clinical } \\
\text { Simulation in } \\
\text { Nursing }\end{array}$ & 2012 & I & $\begin{array}{l}\text { Timothy } \\
\text { Clapper, } \\
\text { Mei Kong }\end{array}$ & $\begin{array}{l}\text { Explorar los principales } \\
\text { componentes de administración } \\
\text { del equipo dos recursos y } \\
\text { principios de factores humanos } \\
\text { y cómo ellos se incardinan en el } \\
\text { trabajo en el equipo en la más } \\
\text { completa herramienta conocida } \\
\text { como TeamSTEPPS } \\
\text { (Estrategias del equipo y } \\
\text { herramientas para mejorar el } \\
\text { desempeño y seguridad del } \\
\text { paciente). }\end{array}$ \\
\hline $\mathrm{XV}$ & $\begin{array}{l}\text { Atrial } \\
\text { Fibrillation in } \\
\text { Long Term Care }\end{array}$ & JAMDA & 2012 & I & $\begin{array}{l}\text { Michael W. Rich } \\
\text { MD }\end{array}$ & $\begin{array}{l}\text { Analizar el diagnóstico y } \\
\text { tratamiento de fibrilación atrial } \\
\text { (FA) en cuidados de larga } \\
\text { duración. }\end{array}$ \\
\hline
\end{tabular}




\begin{tabular}{|c|c|c|c|c|c|c|}
\hline XVI & $\begin{array}{l}\text { Reliable and } \\
\text { Variable } \\
\text { Rounder Care } \\
\text { Delivery Model } \\
\text { for Nursing } \\
\text { Assistants and } \\
\text { Patient Care } \\
\text { Technicians }\end{array}$ & Nurse Leader & 2012 & I & $\begin{array}{l}\text { Tamra E. } \\
\text { Minnier; } \\
\text { Katherine } \\
\text { Brownlee; MPM, } \\
\text { Risa Kosko; } \\
\text { Amy Kowinsky; } \\
\text { Susan Christie } \\
\text { Martin; } \\
\text { Maribeth } \\
\text { McLaughlin; } \\
\text { Judith Shovel, } \\
\text { and Jill Young. } \\
\end{array}$ & $\begin{array}{l}\text { Presentar un nuevo modelo de } \\
\text { prestación de cuidados de } \\
\text { enfermería, que tenga el } \\
\text { propósito de garantizar que el } \\
\text { paciente reciba los cuidados } \\
\text { adecuados en el momento } \\
\text { adecuado, todo el tiempo. }\end{array}$ \\
\hline XVII & $\begin{array}{l}\text { Evaluation of } \\
\text { nursing } \\
\text { adherence to a } \\
\text { paper-based } \\
\text { graduated } \\
\text { continuous } \\
\text { intravenous } \\
\text { regular human } \\
\text { insulin infusion } \\
\text { algorithm } \\
\end{array}$ & Nutrition & 2012 & I & $\begin{array}{l}\text { Roland N. } \\
\text { Dickerson; } \\
\text { Jessica } \quad \text { L. } \\
\text { Johnson; George } \\
\text { O. Maish; Gayle } \\
\text { Minard M.D. } \\
\text { Rex O. Brown. }\end{array}$ & $\begin{array}{l}\text { El objetivo de este estudio fue } \\
\text { evaluar la adhesión de } \\
\text { enfermería al algoritmo de } \\
\text { infusión uso continuo de } \\
\text { insulina regular humana } \\
\text { intravenosa (RHI). }\end{array}$ \\
\hline XVIII & $\begin{array}{l}\text { A Leadership } \\
\text { Challenge: Staff } \\
\text { Nurse } \\
\text { Perceptions } \\
\text { After an } \\
\text { Organizational } \\
\text { TeamSTEPPS } \\
\text { Initiative }\end{array}$ & $\begin{array}{lr}\text { JONA } & \text { (The } \\
\text { Journal } & \text { of } \\
\text { nursing } & \\
\text { administration) }\end{array}$ & 2012 & I & \begin{tabular}{lr}
\multicolumn{2}{l}{ Jessica } \\
Kelly & Foltzer; \\
Ramos; & \\
Diane & G. \\
Schwartz; & \\
Diane & J. \\
Ceravolo. &
\end{tabular} & $\begin{array}{l}\text { Medir la percepción de } \\
\text { enfermeros sobre el trabajo en } \\
\text { equipo y el comportamiento en } \\
\text { su ambiente de trabajho }\end{array}$ \\
\hline XIX & $\begin{array}{l}\text { Short hospital } \\
\text { stays and new } \\
\text { demands for } \\
\text { nurse } \\
\text { competencies }\end{array}$ & $\begin{array}{ll}\text { International } \\
\text { Journal of } \\
\text { Nursing } \\
\text { Practice }\end{array}$ & 2012 & I & $\begin{array}{l}\text { Hamström N, } \\
\text { Kankkunen P, } \\
\text { Suominen T, } \\
\text { Meretoja R }\end{array}$ & $\begin{array}{l}\text { Describir el nível de auto- } \\
\text { evaluación de competencia de } \\
\text { las enfermeras finlandesas y de } \\
\text { su acción efectiva en el } \\
\begin{array}{l}\text { contexto de } \\
\text { ambulatorial. }\end{array}\end{array}$ \\
\hline $\mathrm{XX}$ & $\begin{array}{l}\text { Dementia and } \\
\text { Risk of Adverse } \\
\text { Warfarin- } \\
\text { Related Events } \\
\text { in the Nursing } \\
\text { Home Setting }\end{array}$ & $\begin{array}{l}\text { The American } \\
\text { Journal of } \\
\text { Geriatric } \\
\text { Pharmacothera } \\
\text { py }\end{array}$ & 2012 & I & $\begin{array}{lr}\text { Jennifer } & \text { Tjia; } \\
\text { Terry S. } & \text { Field; } \\
\text { Kathleen } & \text { M. } \\
\text { Mazor; Jennifer } \\
\text { L. } & \text { Donovan; } \\
\text { Abir O. Kanaan; } \\
\text { George } & \text { Reed; } \\
\text { Peter } & \text { Doherty; } \\
\text { Leslie } & \text { R. } \\
\text { Harrold; } & \text { and } \\
\text { Jerry } & \text { H. } \\
\text { Gurwitz.; J. Tjia } \\
\text { et al. }\end{array}$ & $\begin{array}{l}\text { Testar la hipóteis de que las } \\
\text { acciones de enfermería cuando } \\
\text { se desarrollan en casa de } \\
\text { personas con demencia y con } \\
\text { tratamiento de warfarina tienen } \\
\text { menor tiempo de incidencia y } \\
\text { ocurrencia de eventos adversos } \\
\text { que en los casos donde no hay } \\
\text { demencia, tras el control de } \\
\text { características de instalaciones } \\
\text { y paciente. }\end{array}$ \\
\hline XXI & $\begin{array}{l}\text { A Trinity of } \\
\text { Engagement } \\
\text { Transforms Care } \\
\text { at UPMC } \\
\text { Shadyside's } 6 \\
\text { Main }\end{array}$ & $\begin{array}{l}\text { Global } \\
\text { Business and } \\
\text { Organizational } \\
\text { Excellence }\end{array}$ & 2012 & I & Irma D’Antonio & $\begin{array}{l}\text { Presentar la evolución de una } \\
\text { unidad de asistencia, conducida } \\
\text { por una asociación que inluya al } \\
\text { director de la unidad, su equipo, } \\
\text { y un especialista formado en el } \\
\text { mejor uso de Transformar } \\
\text { Cuidados a pie de cama } \\
\text { (TCAB) herramientas. }\end{array}$ \\
\hline
\end{tabular}




\begin{tabular}{|c|c|c|c|c|c|c|}
\hline XXII & $\begin{array}{l}\text { Ultrafiltration } \\
\text { rate as a nursing- } \\
\text { sensitive quality } \\
\text { indicator in } \\
\text { haemodialysis }\end{array}$ & $\begin{array}{l}\text { International } \\
\text { Journal of } \\
\text { Nursing } \\
\text { Studies. }\end{array}$ & 2012 & I & $\begin{array}{l}\text { Magnus } \\
\text { Lindberg; Mette } \\
\text { Spliid Ludvigsen }\end{array}$ & 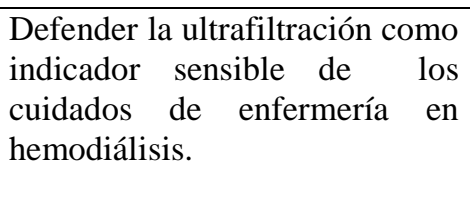 \\
\hline XXIII & $\begin{array}{l}\text { Nurse workload } \\
\text { in implementing } \\
\text { a tight } \\
\text { glycaemic } \\
\text { control protocol } \\
\text { in a UK } \\
\text { hospital: a pilot } \\
\text { time-in-motion } \\
\text { study }\end{array}$ & Nurs Crit Care & 2012 & I & $\begin{array}{l}\text { Juliane } \\
\text { Gartemann, } \\
\text { Elizabeth } \\
\text { Caffrey, Nandini } \\
\text { Hadker, Sheila } \\
\text { Crean, Gary M. } \\
\text { Creed and } \\
\text { Carsten Rausch }\end{array}$ & $\begin{array}{l}\text { Estimar el tiempo acumulado } \\
\text { que los enfermeros de cuidados } \\
\text { críticos utilizan para } \\
\text { implementación de un } \\
\text { protocolo de control glicémico } \\
\text { (TGC) en un estudio (TIM), } \\
\text { realizado en un hospital en } \\
\text { Reino Unido. }\end{array}$ \\
\hline XXIV & $\begin{array}{l}\text { The scope for } \\
\text { replacing } \\
\text { seclusion with } \\
\text { time out in acute } \\
\text { inpatient } \\
\text { psychiatry in } \\
\text { England }\end{array}$ & J Adv Nurs. & 2012 & I & 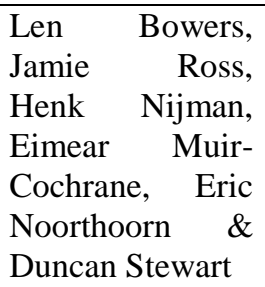 & $\begin{array}{l}\text { Evaluar si la diminución de la } \\
\text { reclusión puede ser una práctica } \\
\text { segura en instituciones } \\
\text { psiquiátricas. }\end{array}$ \\
\hline
\end{tabular}

$E^{1}$ : Espanhol $P^{2}$ : Português I': Inglês

Los resultados presentan la enfermería ligada directamente tanto con la producción del error, como con la promoción de la seguridad. Siendo mencionada también como profesión con mayor probabilidad de identificación del error, debido a las características de su práctica profesional, actuando directamente con el cuidado del paciente en los diversos ámbitos de la asistencia para la salud.

En relación a los ambientes de asistencia para la salud más mencionados entre los estudios que abordan seguridad (Tabla I), se obtuvo que el ambiente hospitalario es citado en 20 (veinte) publicaciones. En tanto que la atención primaria no aparece como escenario de ningun publicación investigada. 
Tabla I: Ambiente de asistencia para la salud que aparece como escenario de discusiónde la temática seguridad. Fortaleza - CE, 2012.

\begin{tabular}{|lcc|}
\hline $\begin{array}{l}\text { Ambiente de asistencia a la } \\
\text { salud }\end{array}$ & $\begin{array}{c}\text { Artículos relacionados } \\
\text { Ambiente hospitalario }\end{array}$ & $\begin{array}{c}\text { Número de } \\
\text { artículos }\end{array}$ \\
& $\begin{array}{c}\mathrm{XI}, \mathrm{II}, \mathrm{II}, \mathrm{IV}, \mathrm{VI}, \mathrm{XIII}, \mathrm{XIV}, \mathrm{XV}, \mathrm{XVI}, \\
\mathrm{XVII}, \mathrm{XVIII}, \mathrm{XIX}, \mathrm{XXI}, \mathrm{XXII}, \\
\mathrm{XXIII},\end{array}$ & \\
\hline $\begin{array}{l}\text { Ambiente de atención } \\
\text { psiquiátrica }\end{array}$ & $\mathrm{XXIV}$ & 1 \\
\hline Refugio de ancianos & $\mathrm{XX}$ & 1 \\
\hline Atención primaria en salud & & - \\
\hline No identificado & $\mathrm{V}, \mathrm{X}$ & 20 \\
\hline Total & - & 24 \\
\hline
\end{tabular}

Se procuró también identificar entre las áreas de saud que forman parte del cuidado de enfermería, como salud del niño, mujer, anciano o mental, aquellas que más están siendo exploradas al discutir la temática seguidad. Obteniéndose que la mayoría de los estudios no identificaron ninguna de las áreas mencionadas anteriormente. $Y$ entre aquellas en que la identificación ocurría, el área más evidente fue la de salud del niño (Tabla II).

Tabla II: Área de actuación del cuidado de enfermería para la salud discutida con la temática seguridad. Fortaleza - CE, 2012.

\begin{tabular}{|lll|}
\hline Salud del Niño & $\mathrm{II}, \mathrm{IV}, \mathrm{VI}, \mathrm{VII}, \mathrm{XII}$ & 5 \\
\hline Salud de la Mujer & $\mathrm{V}, \mathrm{VIII}, \mathrm{X}$ & 3 \\
\hline Salud del Anciano & $\mathrm{XV}, \mathrm{XX}$ & 2 \\
\hline Sslud Mental & $\mathrm{XXIV}$ & 1 \\
\hline No especificado & $\mathrm{I}, \mathrm{III}, \mathrm{IX}, \mathrm{XIII}, \mathrm{IX}, \mathrm{XIV}, \mathrm{XVI}, \mathrm{13}$ \\
& $\mathrm{XVII}, \mathrm{XVIII}, \mathrm{XIX}, \mathrm{XXI}$, \\
& $\mathrm{XXII}, \mathrm{XXIII}$ & $\mathbf{2 4}$ \\
\hline Total & - & \\
\hline
\end{tabular}

En la lectura de los textos, se identificaron los factores que estaban relacionados con el compromiso de la seguridad y otros relacionados como promotores de seguridad (Cuadro 2). Entre los acontecimientos que ponen en peligro la seguridad, los más citados fueron: fallo en la comunicación oral o escrita entre profesionales del equipo y entre profesional y paciente; fallo en la terapéutica medicamentosa, sea en la prescripción, preparación de medicamentos, administración, como en los eventos adversos causados por el uso; fallo en la realización de procedimientos de salud, como transfusión sanguínea, procedimiento perioperatorios, hemodiálisia y terapia de calor; y factores humanos relacionados con el trabajo, como cannsancio, estrés, falta de motivación, sobrecarga y/o (in)satisfacción y negligencia.

Los eventos más citados como promotores de seguridad fueron: cuidado centrado en el paciente/familia, comunicación y diálogo como favoreccedores de las relaciones interpersonales, comunicación abierta entre los profesionales sobre el error; 
promoción de gestión de personas por medio de la práctica de la formación y capacitación de los profesionales; mejora del sistema de prestación de la asistencia sanitaria, por medio de la gestión de la calidad; promoción de políticas de prevención, medición y evaluación de los errores y uniformidad de las prácticas, por medio de la protocolización de los procedimientos.

Cuadro 2: Eventos que ponen en peligro o promueven la seguridad del paciente en el ámbito de los cuidados de enfermería. Fortaleza-CE, 2012.

\begin{tabular}{|c|c|c|c|c|c|}
\hline $\begin{array}{l}\text { Eventos que } \\
\text { comprometen la } \\
\text { seguridad }\end{array}$ & Artículos & $\begin{array}{l}\text { Número } \\
\text { de } \\
\text { artículos }\end{array}$ & $\begin{array}{c}\text { Acciones que } \\
\text { promueven seguridad }\end{array}$ & Artículo & $\begin{array}{l}\text { Número } \\
\text { de } \\
\text { artículos }\end{array}$ \\
\hline $\begin{array}{l}\text { Fallo de recursos } \\
\text { organizacionales y } \\
\text { estructurales }\end{array}$ & $\mathrm{I}, \mathrm{II}, \mathrm{VII}$ & 3 & $\begin{array}{c}\text { Comunicación abierta } \\
\text { sobre el error }\end{array}$ & $\begin{array}{l}\text { XI, XIV, III, } \\
\text { XVIII, XIX }\end{array}$ & 5 \\
\hline $\begin{array}{l}\text { Fallo en la comunicación } \\
\text { oral o escrita entre el } \\
\text { equipo o entre } \\
\text { profesional y paciente }\end{array}$ & $\begin{array}{l}\text { II, XI, XIV, } \\
\text { VIII, XIII }\end{array}$ & 5 & $\begin{array}{c}\text { Detección precoz y } \\
\text { notificación de los } \\
\text { errores y eventos } \\
\text { adversos; }\end{array}$ & $\mathrm{I}, \mathrm{XI}$ & 2 \\
\hline $\begin{array}{l}\text { Fallo em la terapéutica } \\
\text { medicamentosa }\end{array}$ & $\begin{array}{l}\text { XI, II, XIV, XV, } \\
\text { XVII, XX, XXIII }\end{array}$ & 5 & $\begin{array}{c}\text { Exploración de } \\
\text { debilidades que dan } \\
\text { lugar a los eventos } \\
\text { adversos; }\end{array}$ & $\mathrm{I}$ & 1 \\
\hline $\begin{array}{l}\text { Fallos relacionados con } \\
\text { procedimientos de } \\
\text { salud, entre ellos: } \\
\text { transfusión sanguínea, } \\
\text { perioperatorio, } \\
\text { hemodiálisis, terapia de } \\
\text { calor. }\end{array}$ & $\begin{array}{l}\text { I, XIX, XXII, } \\
\text { VI, XIII }\end{array}$ & 5 & $\begin{array}{l}\text { Mejora del sistema de } \\
\text { prestación de asistencia } \\
\text { sanitaria, por medio de } \\
\text { la gestión de la calidad. }\end{array}$ & $\begin{array}{c}\text { I, XIX, XXI, } \\
\text { VII }\end{array}$ & 4 \\
\hline $\begin{array}{l}\text { Fallo de adhesión del } \\
\text { paciente a la terapéutica } \\
\text { prescrita }\end{array}$ & $\mathrm{I}$ & 1 & $\begin{array}{l}\text { Cuidado centrado en el } \\
\text { paciente/cliente y } \\
\text { familia, comunicación, } \\
\text { diálogo y favorecer } \\
\text { relaciones } \\
\text { interpersonales. }\end{array}$ & $\begin{array}{l}\text { XIX, XXI, IV, } \\
\text { V, VII, VIII }\end{array}$ & 6 \\
\hline $\begin{array}{c}\text { Infecciones del ambiente } \\
\text { hospitalario }\end{array}$ & I, IV & 2 & $\begin{array}{l}\text { Promoción de políticas } \\
\text { de prevención, } \\
\text { medición y evaluación } \\
\text { de los errores. }\end{array}$ & I, II, III, XIII & 4 \\
\hline Factores ambientales & $\mathrm{I}$ & 1 & Ambiente no punitivo & XI, XVIII, XIII & 3 \\
\hline $\begin{array}{l}\text { Estado de salud del } \\
\text { paciente }\end{array}$ & $\mathrm{I}, \mathrm{X}$ & 2 & $\begin{array}{l}\text { Gestión de Prsonas y } \\
\text { Formación } \\
\text { complementaria }\end{array}$ & $\begin{array}{l}\text { XIV, XXI, V, } \\
\text { VII, XII }\end{array}$ & 5 \\
\hline Jerarquización rígida & $\overline{X I V}$ & 1 & $\begin{array}{l}\text { Promoción de } \\
\text { clima/cultura de } \\
\text { seguridad }\end{array}$ & III, XIII & 2 \\
\hline Riesgo de caída & IX & 1 & $\begin{array}{l}\text { Gestión con } \\
\text { compromiso de } \\
\text { seguridad }\end{array}$ & III, XVIII & 2 \\
\hline Aislamiento social & $\overline{X X I V}$ & 1 & Ergonomía & XIV & 1 \\
\hline Cultura de productividad & $\mathrm{VII}$ & 1 & Trabajo en equipo & $\mathrm{XVIII,} \mathrm{XXI}$ & 2 \\
\hline Falta de conocimiento & XIII & 1 & $\begin{array}{c}\text { Uniformidad de las } \\
\text { prácticas, } \\
\text { establecimiento de } \\
\text { protocolos }\end{array}$ & $\begin{array}{c}\text { XVI, XVII, } \\
\text { XXIII, VII, XIII }\end{array}$ & 5 \\
\hline $\begin{array}{c}\text { Factores humanos } \\
\text { (cansancio, estrés, falta } \\
\text { de motivación, } \\
\text { sobrecarga y/o } \\
\text { (in)satisfacción y } \\
\text { negligencia) }\end{array}$ & $\begin{array}{l}\text { I, II, XIV, VII, } \\
\text { VIII, XIII }\end{array}$ & 5 & & & \\
\hline
\end{tabular}




\section{SEGURIDAD Y SU DIVERSIDAD CONCEPTUAL}

El término seguridad viene siendo discutido en el contexto de la asistencia a la salud, debido a la elevación de la complejidad de las prácticas clínicas y de las organizaciones de salud, siendo un tema motivo de análisis, diálogo y reflexión ${ }^{(6,7)}$.

La seguridad es un fenómeno singular, que depende de la percepción, interpretación y de los proyectos de vida de cada persona. Un ambiente puede parecer seguro para profesionales $y$, a la vez, puede presentarse inseguro para el paciente, cuando la comunicación, el vínculo y el diálogo no se establecen entre las partes. Por esto, el ver y el escuchar son fundamentales para la construcción de una práctica segura ${ }^{(8)}$. Quien está siendo asistido tiene que tener la oportunidad de ser sentido, escuchado y comprendido para el establecimiento de una relación de confianza entre quien cuida y quien está siendo cuidado.

La promoción de la seguridad en la salud proporciona relación directa con la calidad de la asistencia. Autores definen seguridad como la ausencia de daños innecesarios, reales o potenciales producidos por el proceso de asistencia para la salud, así como por las estrategias destinadas a prevenir o minimizar los daños ${ }^{(3,6,9) \text {. }}$

Cuando se busca comprender seguridad a partir de la escala de la jerarquía de las necesidades, propuestas por Abraham $\mathrm{H}$. Maslow, la seguridad aparece como segunda necesidad de jerarquía, debiendo satisfacerse cuando la de orden inferior, necesidades fisiológicas, haya sido atendidas, para que así las necesidades jerarquícamente superiores en la escala, como amor/relación, estima y realización personal, puedan ser satisfechas ${ }^{(7)}$.

La necesidad de sentirnos seguros es una preocupación que dura toda la vida. Y de acuerdo con la escala de Maslow, son necesidades de seguridad: la dependencia, protección, estabilidad, ausencia de miedo, ansiedad o caos, necesidad de estructura, límites, órdenes, leyes, entre otras. Todas estas necesidades satisfechas orientan para evitar 0 , incluso, neutralizar las situacionees de peligro ${ }^{(7)}$.

Sin embargo, incluso si todos los riesgos de ocurrencia de errores pudieran medirse, si el diálogo y la comunicación entre las partes no hubieran sido establecidas, la seguridad de la asistencia puede aún estar siendo amenazada.

En el contexto de las práticas de salud, el aumento de la complejidad de las prácticas clínicas, asociada a las incertidumbres de las tomas de decisiones, incremento tecnológico y mayor uso de los servicios de salud hizo que el tema seguridad fuese cada vez más trabajado en la literatura debido a la posibilidad y, hasta incluso, ocurrencia de eventos adversos derivados del proceso asistencial ${ }^{(4,10)}$.

La Organización Mundial de la Salud define evento adverso como aquel incidente que causa daños a la salud del paciente, relacionado con la asistencia sanitaria ${ }^{(11) .}$

Así, la temática seguridad se traduce como prioridad en las estrategias de salud ${ }^{(6)}$. Y la enfermería como profesión actuante en la asistencia a la salud también es bastante debatida en las literaturas que discuten dicha temática. 
Autores mencionan a la enfermería tanto como contribuyente en la producción del error ${ }^{(12-14)}$, como también en la promoción de la seguridad (13-16), por actuar directamente en la práctica del cuidado con el paciente.

La enfermería está directamente relacionada con la realización de eventos que están asociados a la ocurrencia de errores, como la administración de medicamentos y realización de procedimientos terapéuticos de la prática en salud.

Por tanto, la práctica de la enfermería debe estar centrada en el cuidado, con respaldo del conocimiento, en el diálogo y en el establecimiento de relaciones interpersonales satisfactorias, y basada en actitudes y habilidades en la promoción de un ambiente seguro, al poder promover cuidados sin que haya ocurrencia de daños $y$, por ello, una sensación de seguridad $(7,17-19)$.

Como cuidadores de salud, los profesionales están llamados a apreciar la calidad del servicio que ofrecen ${ }^{(7)}$. $\mathrm{Y}$, estando esa calidad asociada a la seguridad, esta pasa a ser estrategia esencial para la excelencia del cuidado a ser prestado.

Así se percibe en el presente estudio, por medio de la Tabla I, que la literatura también preconiza que el término seguridad se aborda predominantemente en la atención hospitalaria, mientras que la atención primaria tiene un reducido número de estudios. Esta característica es compatible con los resultados de la literatura que justifican la elevación de los estudios en la atención hospitalaria por el aumento del número de aparatos tecnológicos y la identificación más clara de la ocurrencia de errores. Hecho que no anula la ocurrencia del error en la atençión primaria, pues en ese ámbito los estudios y la busca de la seguridad también se justifican ${ }^{(9,13)}$.

\section{LÍNEAS DE CUIDADO Y SOLVENCIA ASISTENCIAL}

En los estudios consultados se observa que la asistencia de enfermería discute las líneas de cuidado salud del niño, salud de la mujer, salud del anciano y salud mental. Estas líneas en sus varias dimensiones y área de actuación.

El área de actuación más trabajada en el contexto de la seguridad fue la salud del niño (Tabla II), siendo abordadas las cuestiones relacionadas con el proceso de administración de medicamentos; realización de procedimientos terapéuticos como la terapia de calor y el empleo de ultrasonografía para promoción de la seguridad en el procedimiento de punción intravascular periférica en niños; valorización de la presencia de la familia en el cuidado al niño, como factor promotor de seguridad y satisfacción del paciente; y comprensión del niño acerca de la seguridad del cuidado de enfermería ${ }^{(12,14,16,20,21)}$.

En los estudios que abordaban a la mujer como sujeto de estudio, se pudo investigar la temática seguridad basándose en las vivencias anteriores que las mujeres vivieron a lo largo de su vida, como eventos de abuso sexual en la infancia y su relación con la percepción de (in)seguridad en el proceso de contacto perinatal para sí y para el bebé; percepción interpretativa de la seguridad de mujeres que relataban inseguridad durante el parto; y análisis de comportamientos de mujeres con cáncer de mama que habian iniciado el amamantamiento, en relación al proceso de seguridad ${ }^{(8,17,22) \text {. }}$

En la investigación con mujeres, se percibió la importancia de proporcionar escucha al paciente, entendiéndolo como aquel que vivencia el evento, por eso, muchas veces 
no presenta lenguaje para comunicar el error. El fallo en esa disponibilidad de escucha puede proceder, según el autor, de la falta de tiempo y estrés de los profesionales de salud, causando negligencia en las relaciones y fragilidad en la integridad relacional ${ }^{(8)}$.

La escucha debe ocurrir de manera cualificada y siempre con el compromiso de acogerse a las necesidades de los ciudadanos que procuran los servicios de salud ${ }^{(23)}$, proporcionando seguridad y establecimiento de vínculos que fortalezcan la relación profesional y usuario.

Esta relación hace imprescindible la promoción de una comunicación eficaz tanto entre profesionales, como entre estos y los usuarios del sistema de salud. El fallo en la comunicación comprende también un factor de contribución para ocurrencia del error, que puede ser expresado por la lectura errada del nombre del medicamento, escrito de modo ilegible en la prescripción, comunicación gráfica en equipamientos hospitalarios no comprensibles ${ }^{(24)}$.

En los estudios en que el cuidado al anciano fue abordado, se enfatizó la práctica de cuidado relacionada con la terapéutica medicamentosa ${ }^{(25,26)}$.

Esta terapéutica se identificó como uno de los eventos más relacionados con el comprometimiento de la seguridad. $Y$ cuando se realiza en la práctica geriátrica, predispone a mayores riesgos relacionados tanto con la práctica de multifármacos, como de aparición de efectos adversos. Estando el error de medicación caracterizado como un evento evitable, ocurrido por el uso del medicamento, que puede causar daño o no al paciente ${ }^{(19,26-29)}$. Se trata de un procedimiento rutinariamente realizado por la enfermería, exigiendo, para su práctica, de conocimiento científico y técnico que posibilite el desarrollo de prácticas seguras ${ }^{(12)}$.

La práctica de la terapia medicamentosa comprende desde la prescripción hasta la administración del medicamento, estando implicados en la realización de este proceso diversos profesionales, como médicos, farmacéuticos y equipo de enfermería. Sin embargo, es en esta última categoria profesional, que cuida del procedimiento de administración, en quien recae la mayor responsabilidad sobre el error ${ }^{(12)}$, debido al hecho del contacto directo con el paciente y con la manifestación del error.

Lo que justifica, para algunos autores, la realización de la estandarización y uniformidad de las prácticas en el intento de minimizar las chances de errores y la realización de técnicas por imprevisibilidad ${ }^{(16,28-32)}$

El fallo en la comunicación contribuye a la situación del error, siendo que su práctica, cuando realizada de forma satisfactoria, puede ayudar en la promoción y mantenimiento de un ambiente seguro. Esta comunicación es facilitada cuando se da en la realización de un trabajo en equipo, con diálogo abierto acerca del error ${ }^{(18,19) \text {. }}$

Actualmente, la discusión abierta sobre el error es una práctica largamente reforzada como movimiento en pro de la seguridad del paciente. Sin embargo, la cultura del silencio aún persiste en la realidad de la mayoría de las instancias de salud, lo que resulta en la pérdida de la oportunidad de aprendizaje a partir del error, y así, dificulta la promoción de seguridad ${ }^{(19)}$. 
Villarreal Cantillo ${ }^{(7)}$ resalta que es "fundamental centrarse en la busca de la explicación de cómo y por qué fallaron los mecanismos de defensa y no buscar culpables". Es en esa búsqueda de la explicación de cómo y por qué fallaron y en la tentativa de prevenir nuevos errores, que el autor destaca algunas acciones de seguridad, como: promocion de sistema de notificación de eventos adversos; capacidad de anticiparse a los errores y explorar las debilidades que dan lugar a los eventos adversos; y mejora del sistema de prestación de asistencia sanitaria, para reconfigurar estructuras y reajustar incentivos.

La notificación del error se trata de un relato detallado escrito por los profesionales involucrados acerca de las circunstancias que rodean a este. Esta notificación sólo se torna real cuando se da la cultura del diálogo abierto sobre el error, donde no se prioriza la identificación de los culpables y, así, la busca de la mejora del cuidado y prevención de la ocurrencia de nuevos fallos, que pueden derivarse tanto del hombre, como de la complejidad del sistema en cuestión ${ }^{(19) .}$

Los profesionales deben mantenerse vigilantes para prevenir erores, para detectar precozmente los fallos y flaquezas del sistema de asistencia a la salud ${ }^{(32)}$, que puede estar tanto implicado en las cuestiones de la tecnología dura, como en la tecnología de las relaciones ${ }^{(33)}$.

Sin embargo, otras prácticas de cuidado también pueden estar relacionadas con la promoción de (in)seguridad, como evalúa ${ }^{(34)}$ que relaciona seguridad con el auge de la práctica de disminuir la reclusión en instituciones psiquiátricas, presentando que hay buenas evidencias de que esta práctica puede darse de forma segura..

Garantizar una atención segura y eficiente de los pacientes es uno de los elementosclave de la práctica de enfermería, conquistada por medio de la práctica del cuidado centrado en el paciente, del trabajo en equipo, de la formación y capacitación de los profesionales involucrados, valorización del diálogo y de las relaciones. Los enfermeros, por tanto, precisan mantener una buena comunicación, habilidades de coordinación para mantener la dignidad del paciente y establecer relaciones interpersonales tanto con el paciente como con los demás miembros del equipo profesional, garantizando, así, una mayor satisfacción de los pacientes en relación con los cuidados recibidos y una mejor calidad y seguridad del cuidado ofrecido $(15,16,35)$.

Para esto, uno de los elementos que precisa ser enfrentado es la cultura de la organización de los servicios de salud en pro de la producción, que muchas veces se configura en detrimento de la calidad; así como el enfrentamiento contra la sobrecarga de trabajo, jerarquización de la asistencia, negligencia de las prácticas, lo que puede llevar a la generación de fatiga, estrés, cansancio y, así, poner en peligro la seguridad y la calidad del cuidado ${ }^{(8,16,24)}$.

Es necesaria la implantación de la cultura de seguridad entre professionales, gestores y pacientes, con el desarrollo de un ambiente no-punitivo, que focaliza en la busca de la mejora de las prácticas, identificación de los fallos del sistema, compartir los resultados y co-participación en el cuidado ${ }^{(19,27,13)}$. 


\section{CONCLUSIONES}

Las prácticas de salud pueden estar relacionadas con la generación de riesgos para la salud de los individuos y, así, comprometer la seguridad y la calidad del proceso de asistencia para la salud.

La enfermería desempeña un papel crucial en la promoción de la seguridad del paciente por presentarse de forma más constante y directa en el cuidado al paciente. En contrapartida, si este cuidado no se realiza con calidad puede generar errores y poner en peligro la seguridad del cuidado.

El estudio presentó la práctica de enfermería ligada a las líneas de cuidado al niño, mujer, anciano y en el área de salud mental, teniendo en cada una un abordaje diferenciado de las prácticas de cuidado seguro, tanto en la forma más tradicionalmente abordada, como es el caso de las prácticas medicamentosas, como en el abordaje de la línea hermenéutica, donde el cuidado trasciende la dimensión de promoción de instrumentales y recursos humanos favorables y asume una comprensión más singular del sujeto que está siendo cuidado. Hecho que evidencia una renovación en los abordajes sobre la seguridad.

Las prácticas seguras de salud deben ser incentivadas con la promoción de un trabajo en equipo, valorización del diálogo, de la comunicación abierta sobre el error, vigilancia para detección precoz de los fallos, promoción de ambientes que valoren al trabajador, disminuyendo las consecuencias sobrevenidas de la sobrecarga de trabajo y generación de estrés, cansancio y desmotivación. Así como el desarrollo de un cuidado centrado en el paciente y en la familia, favorecimiento de las relaciones interpersonales, mejora del sistema de prestación de la asistencia sanitaria, por medio de la gestión de la calidad y promoción de políticas de prevención de errores y promoción de seguridad y calidad del proceso asistencial.

\section{REFERENCIAS}

(1). Campbell, S. M.; Roland, M. O.; Buetow, S. A. Defining quality of care. Soc Sci Med, v. 51, n. 11, p. 1611-25, Dec, 2000.

(2). Racoveanu, N.T.; Johaanses, K.S. Tecnologia para El mejoramiento contínuo de La calidad de La atención sanitária. Foro Mundial de La Salud. v. 16, p. 158-65, 1995.

(3). Terol E, Agra Y. Seguridad y Calidad. In: Aranaz J.M. et al. Gestión sanitaria. Calidad y seguridad de lós pacientes. Madrid: Díaz de Santos; 2008.

(4). Prieto Rodriguez, M. A. et al. [Patient perceptions on healthcare safety]. Med Clin (Barc), v. 131 Suppl 3, p. 33-8, Dec, 2008.

(5). Aranaz, J. M. et al. [Health assistance as a risk factor: side effects related to clinicalpractice]. Gac Sanit, v. 20 Suppl 1, p. 41-7, Mar, 2006.

(6). Tejedor Fernández, M. Programas de seguridad del paciente en las unidades de tratamiento del dolor. Sevilla: Agencia de Evaluación de Tecnologías Sanitarias de Andalucía, 2011. $79 \mathrm{p}$.

(7). VillarreaL Cantillo, E. Seguridad de los pacientes. Un compromiso de todos para un cuidado de calidad. Salud, Barranquilla [online]. v.23, n.1, p. 112-119, 2007.

(8). Smythe, E. Safety is an interpretive act: a hermeneutic analysis of care in childbirth. Int J Nurs Stud, v. 47, n. 12, p. 1474-82, 2010.

(9). Saturno, P. J. Seguridad del paciente en atención primaria: marco conceptual, importancia y estratégias de prevención. Universidad de Murcia. 3o Congresso 
Andaluz de Medicos de Atención Primaria Mesa Redonda: Seguridad del Paciente. Uma nueva estrategia de calidad, 2008.

(10). Aranaz, J. M. et al. [Adverse effects in hospital healthcare. A critical review]. Med Clin (Barc), v. 123, n. 1, p. 21-5, Jun, 2004.

(11). WHO, World Health Organizatio. Marco conceptual de la Clasificación Internacional para La Seguridad del Paciente. Versión 1.1. Informe Técnico Definitivo. Ginebra, 2009.

(12). Yamanaka, T. I. et al. Redesenho das atividades de enfermagem para redução de erros de medicação em pediatria. Rev. bras. enferm. [online]. v.60, n.2, pp. 190196, 2007.

(13). Hughes, L. C.; Chang, Y.; Mark, B. A. Quality and strength of patient safety climate on medical-surgical units. Health Care Manage Rev, v. 34, n. 1, p. 19-28, JanMar 2009.

(14). Brady, M. Hospitalized children's views of the good nurse. Nurs Ethics, v. 16, n. 5, p. 543-60, Sep 2009.

(15). Hamstrom, N. et al. Short hospital stays and new demands for nurse competencies. Int J Nurs Pract, v. 18, n. 5, p. 501-8, Oct, 2012.

(16). Wilson, B. L. Keeping an eye on patient safety using human factors engineering (HFE): a family. J Spec Pediatr Nurs, v. 15, n. 1, p. 84-7, Jan 2010.

(17). Coles, J.; Jones, K. "Universal Precautions": perinatal touch and examination after childhood sexual. Birth, v. 36, n. 3, p. 230-6, Sep 2009.

(18). Castner, J. et al. A leadership challenge: staff nurse perceptions after an organizational. J Nurs Adm, v. 42, n. 10, p. 467-72, Oct 2012.

(19). Belela, A. S. C.; Peterlini, M. A. S.; Pedreira, M. da L. G. Revelação da ocorrência de erro de medicação em unidade de cuidados intensivos pediátricos. Rev. bras. ter. intensiva, São Paulo, v. 22, n. 3, Sept. 2010.

(20). Lane, E.; Latham, T. Managing pain using heat and cold therapy. Paediatr Nurs, v. 21, n. 6, p. 14-8, Jul 2009.

(21). Avelar, A. F. M. et al. Capacitação de enfermeiros para uso da ultrassonografia na punção intravascular periférica. Acta paul. enferm. [online]. v.23, n.3, p. 4334362010.

(22). Azim, H. A., JR. et al. Breastfeeding in breast cancer survivors: pattern, behaviour and effect on breast. Breast, v. 19, n. 6, p. 527-31, Dec, 2010.

(23). BRASIL, Ministério da Saúde. Secretaria de Atenção à Saúde. Núcleo Técnico da Política Nacional de Humanização. HumanizaSUS: Documento base para gestores e trabalhadores do SUS. 4. ed. 4. reimp. - Brasília : Editora do Ministério da Saúde, 2010, 72 p.

(24). Clapper, T. C.; Kong, M. TeamSTEPPS®: The Patient Safety Tool That Needs to Be Implemented. Clinical Simulation in Nursing, v. 8, n. 8, p. e367-e373, 2012.

(25). Rich, M. W. Atrial fibrillation in long term care. J Am Med Dir Assoc, v. 13, n. 8, p. 688-91, Oct 2012.

(26). Tjia, J. et al. Dementia and risk of adverse warfarin-related events in the nursing home setting. Am J Geriatr Pharmacother, v. 10, n. 5, p. 323-30, Oct , 2012.

(27). Otero P, Leyton A, Mariani G, Ceriani Cernadas JM;Patient Safety Committee. Medication errors in pediatric inpatients: prevalence and results of a prevention program. Pediatrics. 2008;122(3):e737-43.

(28). Gartemann, J. et al. Nurse workload in implementing a tight glycaemic control protocol in a UK. Nurs Crit Care, v. 17, n. 6, p. 279-84, Nov, 2012.

(29). Nóbrega, O. de T. N.; Karnikowski, M. G. de O. A terapia medicamentosa no idoso: cuidados na medicação. Ciência e Saúde Coletiva. v.10, n2, p. 309-313, 205. 
(30). Minnier, T. E. et al. Reliable and Variable Rounder Care Delivery Model for Nursing Assistants and Patient Care Technicians. Nurse Leader, v. 10, n. 5, p. 28-35, 2012.

(31). Dickerson, R. N. et al. Evaluation of nursing adherence to a paper-based graduated continuous intravenous. Nutrition, v. 28, n. 10, p. 1008-11, Oct, 2012.

(32). Odom-Forren, J. The normalization of deviance: a threat to patient safety. J Perianesth Nurs, v. 26, n. 3, p. 216-9, Jun 2011.

(33). Merhy, E. E. et al. O trabalho em saúde: olhando e experienciando o SUS no cotidiano. 3a Ed. São Paulo: Editora Hucitec, 2007. 296 p.

(34). Bowers, L. et al. The scope for replacing seclusion with time out in acute inpatient psychiatry in. J Adv Nurs, v. 68, n. 4, p. 826-35, Apr, 2012.

(35). D'Antonio, I. A trinity of engagement transforms care at UPMC Shadyside's 6 Main.2012. 